Article

\title{
Carotenoid-Chlorophyll Interactions in a Photosynthetic Antenna Protein: A Supramolecular QM/MM Approach
}

\author{
Matthew J. Guberman-Pfeffer and José A. Gascón * \\ Department of Chemistry, University of Connecticut, Storrs, CT 06269-3060, USA; \\ matthew.guberman-pfeffer@uconn.edu \\ * Correspondence: jose.gascon@uconn.edu; Tel.: +1-860-486-0591
}

This paper is published as part of a thematic issue of Molecules on "Combined Quantum Mechanical and Molecular Mechanical Methods and Simulations".

(http://www.mdpi.com/journal/molecules/special_issues/QM)

\begin{abstract}
Multichromophoric interactions control the initial events of energy capture and transfer in the light harvesting peridinin-chlorophyll $a$ protein (PCP) from marine algae dinoflagellates. Due to the van der Waals association of the carotenoid peridinin (Per) with chlorophyll $a$ in a unique 4:1 stoichiometric ratio, supramolecular quantum mechanical/molecular mechanical (QM/MM) calculations are essential to accurately describe structure, spectroscopy, and electronic coupling. We show that, by enabling inter-chromophore electronic coupling, substantial effects arise in the nature of the transition dipole moment and the absorption spectrum. We further hypothesize that inter-protein domain Per-Per interactions are not negligible, and are needed to explain the experimental reconstruction features of the spectrum in wild-type PCP.
\end{abstract}

Keywords: supramolecular QM/MM; PCP; peridinin; chlorophyll $a$; light harvesting

\section{Introduction}

The capture, conversion, and transfer of solar energy by artificial means constitute some of the greatest research challenges at present [1,2]. Natural architectures that overcome these difficulties have been evolved by photosynthetic algae, bacteria, and plants, which collectively consume an estimated five times more power than is needed for all human activities [3]. Lessons learned from the mechanistic study of natural light harvesting complexes will inform and inspire the rational design of artificial photosynthetic devices capable of meeting growing energy demands for sustainable human development $[1,4,5]$.

A notable example of a highly efficient light harvesting antenna is the water-soluble peridinin-chlorophyll $a$ protein (PCP) from marine algae dinoflagellates [6]. In the main form of PCP (MFPCP), isolated from Amphidinium carterae [7], the complex is characterized by a $\mathrm{C}_{2}$-pseudosymmetric, two domain $\alpha$-helical protein capsule of densely packed chromophoric assemblies. Each cofactor cluster has a unique 4:1 stoichiometric ratio of the structurally distinctive carotenoid peridinin (Per) and chlorophyll $a(\mathrm{Chl} a)$, as seen in Figure 1a, as well as a molecule of the lipid digalactosyldiacylglycerol. Per is a heptene-based chromophore that features a $\mathrm{C}_{37}$, instead of the standard $\mathrm{C}_{40}$ carotenoid framework, as well as an unusual pairing of allenic and $\gamma$-butenolide moieties, as seen in Figure 1b, which significantly shapes the photophysical response of the polyene chain to the polarity, proticity, and polarizability of the local environment [6]. 
A reconstituted, recombinant PCP (RFPCP) complex (Figure 1c), which is a homodimer of the $\mathrm{N}$-terminal domain from the wild-type protein, has been used as a model system to study both spectral tuning and excitation energy transfer [4]. The cofactor clusters in either MF- or RFPCP harvest blue-green photons with $\sim 90 \%$ efficiency [4] by mechanisms intimately related to the structure of Per and the architecture of the solvated PCP complex [8].

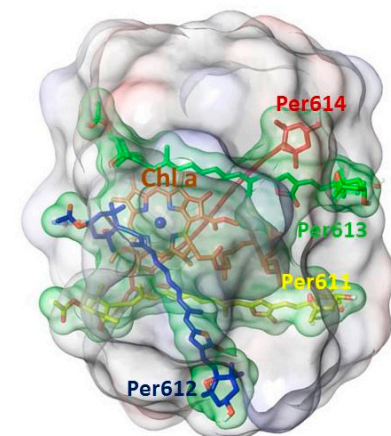

(a)

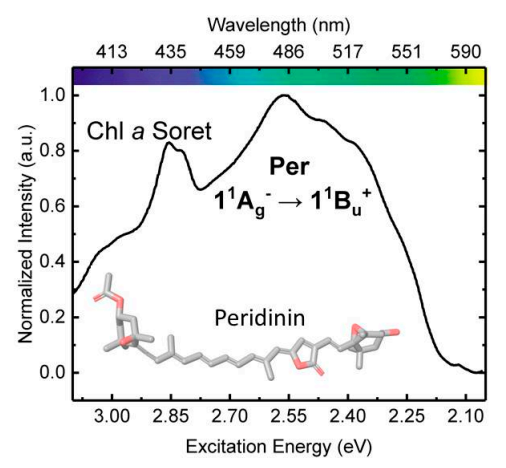

(b)

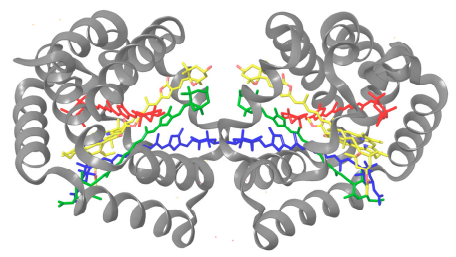

(c)

Figure 1. (a) Partial view of the cofactor cluster inside a single domain of the reconstituted, recombinant peridinin-chlorophyll $a$ protein (RFPCP) complex. Highlighted in green is the supramolecular assembly consisting of four bound peridinin (Per) molecules and one chlorophyll $a$ (Chl $a$ ) encapsulated by the van der Waals surface of the protein. (b) Absorption spectrum of the peridinin-chlorophyll a protein (PCP) complex (ref. [4]) and chemical structure of Per. (c) Full view of the RFPCP $\mathrm{C}_{2}$-symmetric homodimer.

The linear absorption spectrum of Per is commonly interpreted using a three-state model composed of the ground $\left(\mathrm{S}_{0}\right)$ and first two excited singlet states $\left(\mathrm{S}_{1}\right.$ and $\left.\mathrm{S}_{2}\right)$. In analogy to a rigorously

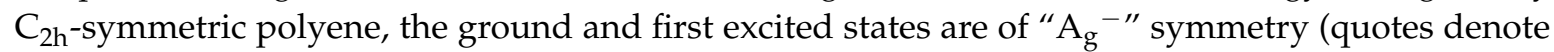
"approximate"), and one-photon transitions between these states are forbidden by symmetry selection rules [9]. Though the substitution pattern of the polyene chain in Per strongly breaks $C_{2 h}$ molecular symmetry, the $\mathrm{S}_{0} \rightarrow \mathrm{S}_{1}$ transition is still not observed experimentally. Fiedor et al. [10] have proposed an alternate explanation for the forbidden character of this transition which involves an unfavorable Frank-Condon overlap of the $S_{0}$ and $S_{1}$ potential energy surfaces due to severe geometric distortions in the $S_{1}$ state. The second excited state has " $\mathrm{B}_{\mathrm{u}}{ }^{+\prime}$ symmetry. The $\mathrm{S}_{0}\left({ }^{\prime \prime} 1^{1} \mathrm{~A}_{\mathrm{g}}{ }^{-\prime}\right) \rightarrow \mathrm{S}_{2}\left({ }^{\prime \prime} 1^{1} \mathrm{~B}_{\mathrm{u}}{ }^{+\prime}\right)$ excitation is strongly one-photon allowed, dominates the optical spectrum in the $450-550 \mathrm{~nm}$ region, and represents the initial step of light harvesting by PCP. Once energy is collected by the Per spectral forms, it is transferred to the Qx and Qy states of $\mathrm{Chl} a$, and ultimately shuttled into Photosystem II. The Qx and Qy states (600-700 nm) are the two lowest-lying excited states of Chl $a$, with Qx being the closest in energy to the most red-shifted absorber among the Pers $(\sim 510 \mathrm{~nm})$. Qy is dominated by $\mathrm{HOMO} \rightarrow$ LUMO and (HOMO -1$) \rightarrow(\mathrm{LUMO}+1)$ configurations, whereas Qx is primarily $(\mathrm{HOMO}-1) \rightarrow$ LUMO and $\mathrm{HOMO} \rightarrow(\mathrm{LUMO}+1)$ in character.

The $S_{2}$ state of the four Pers within a functional unit of PCP are tuned to absorb at specific wavelengths distributed over a $58 \mathrm{~nm}$ range of maximal solar irradiance [4]. By contrast, the maximal solvatochromic shift reported for the $\mathrm{S}_{2}$ absorption maximum in a variety of homogeneous solvents is only 16-28 nm [6]. We recently reported multichromophoric quantum mechanical/molecular mechanical (QM/MM) simulations that delineated the controlling factors for wavelength regulation in PCP, and permitted the first unambiguous assignment of the distinct Per spectral signatures to specific chromophores [8]. Protein-induced conformational distortion, inter-chromophore electronic coupling, and the electrostatic environment of the complex, mediated by an aqueous dielectric, were found to make nearly equal and additive contributions to the $S_{0} \rightarrow S_{2}$ wavelength variation. These supramolecular QM/MM simulations also identified molecular excitons delocalized over two or three chromophores depending on the absorption wavelength, thereby directly addressing a long-standing debate over the presence and relevance of exciton delocalization on PCP optical properties [11-13]. 
Recent theoretical [14,15] and experimental [15-17] work has identified an ultrafast quantum coherent mechanism in the Per-to-Chl $a$ energy transduction process. While it is now well established that Per-to-Chl $a$ energy transfer represents a fundamental event in light harvesting by PCP, it is still unknown if energy transfer paths among Pers, and from multiple Pers to Chl $a$, help funnel the transfer of energy. A step towards such understanding would at least require a full characterization of electronic coupling strengths between all chromophoric cofactors. It is only recently, with the aid of supramolecular QM/MM approaches [8], that such evaluations can be accurately obtained. Supramolecular QM/MM refers to the implementation of QM/MM methods where the quantum mechanical $(\mathrm{QM})$ regions involve entire supramolecular assemblies of distinct units. These units exhibit subtle interactions controlling structure, function and spectroscopy. In this report, we characterize the excitonic interactions in PCP to describe the strengths and environmental regulation of inter-chromophore electronic coupling. Our findings add to the detailed understanding of light harvesting strategies operative in this exceptionally efficient protein antenna complex.

\section{Materials and Methods}

\subsection{Model Preparation}

A model of the reconstituted PCP complex from the highest resolution crystal structure available (PDB ID 3IIS; $1.4 \AA$ ) [4] was prepared. The A and M chains, which non-covalently associate to form a $\mathrm{C}_{2}$-symmetric homodimer of the wild-type PCP N-terminal domain, were retained. Because of the symmetry, preparation decisions described for one subunit apply to both domains. Unless otherwise noted, QM regions were applied on the $\mathrm{M}$ chain pigments in the presence of the full homodimer. The reconstituted complex strongly resembles structurally and spectroscopically wild-type PCP $[4,18]$. Experimental spectral reconstructions for both complexes found four 0-0 bands that agree within $8 \mathrm{~nm}[4,18]$. Three of these features correspond to absorption by two Pers that are presumably related by the $C_{2}$ symmetry of the complex, whereas the fourth spectral origin is attributed to a single Per. A fifth spectral origin due to a single Per is only observed for the wild-type complex. This experimental data strongly suggests that an analysis of reconstituted PCP is transferrable to the wild-type complex, and applies to the spectral tuning of all but one Per in wild-type PCP.

Hydrogen atoms were added, protonation and tautomeric states assigned, and the H-bonding network optimized by reorienting donor/acceptor side chains, using the Protein Preparation application in Maestro version 10.6.014 [19]. Each chain contains 20 acidic and 18 basic residues (not counting His) that are primarily surface exposed, and these residues were assigned standard protonation states for $\mathrm{pH}$ 7.0. His-66 was assigned the neutral $\mathrm{N}$ tautomeric state, because the other imidazole nitrogen accepts a H-bond from a water molecule coordinated to $\mathrm{Chl} a$. His- 67 was assumed protonated $(\mathrm{His}-\mathrm{H}+)$ following the assignment of Bricker and Lo [14]. For an optimized H-bonding network, the side chains of Asn-24, Gln-30, and Asn-102 were flipped by $180^{\circ}$. All bulk crystallographic waters, which constitute an incomplete and statistically unrepresentative configuration of the solvated state, were removed. To approximate the native environment for the water-soluble complex, an aqueous implicit solvent was modeled with the Poisson-Boltzmann finite difference (PBF) method [20] using the highest resolution grids available. However, a few crystallographic waters were retained, including the water molecule that serves as the fifth coordination to the $\mathrm{Mg}$ of $\mathrm{Chl} a$, and a structural water that participates in a bridging H-bonding network between Tyr-136 and Per613. The solvent accessible surface in the PBF calculations was formed by considering the full homodimer.

\subsection{Structure Optimizations and $Q M / M M$ Simulations}

The four Pers and $\mathrm{Chl} a$ of a cofactor cluster were subjected to torsionally restrained optimizations in the respective binding sites to assess the relevance of experimental coordinate uncertainty. Dihedrals are generally regarded as more reliable from X-ray crystallographic structures than bond lengths and angles [21]. Excitation energies changed by $<60 \mathrm{meV}$ upon optimization for all four Pers and Chl $a$ 
when individually included in the QM region, and therefore we chose to use the X-ray (non-optimized) geometries in all the single or multichromophore QM/MM calculations.

We have shown before that the absorption wavelengths agreed within $5 \mathrm{~nm}$ with or without Chl $a$ added to the QM region, and that the nature of the Per $\mathrm{S}_{0} \rightarrow \mathrm{S}_{2}$ transitions were unaffected. Therefore, the $\mathrm{QM}$ region in all the supramolecular $\mathrm{QM} / \mathrm{MM}$ calculations of this current work only contains Per molecules (up to eight Per molecules in the largest set-up).

All QM/MM simulations, except for the calculation of PBF charges, were performed in Gaussian 16 Rev. B.01 (Gaussian Inc, Wallingford, CT, USA), [22]. The B3LYP/6-31g(d) model chemistry and the Amber99 force field were used for the QM and MM regions, respectively. This methodology was used to calculate electrostatic potential surface (ESP) charges on all non-standard residues, as well as to perform the restrained optimizations. The MM region was converted to a background charge distribution for the inter-pigment electronic interaction analysis described in a following subsection. This step was necessary because the electronic energy transfer (EET) methodology $[23,24]$ in Gaussian 16 uses fragment definitions that currently conflict with the layer specifications needed for QM/MM calculations using the ONIOM method. PBF charges were added to the background charge distribution for excitation calculations to model the effect of solvent on inter-chromophore interactions. PBF charges were obtained from a single point calculation on the RFPCP complex in Qsite [25] from Schrodinger LLC. (New York, NY, USA), in which all four Pers within a cofactor cluster of the complex were included in the QM region. These equilibrated PBF charges were used to represent the solvent reaction field in the Gaussian QM calculations as part of the background charge distribution. The PBF charges were calculated using the B3LYP/LACVP * model chemistry and the OPLS-AA force field for the QM and MM regions, respectively. This Qsite calculation was performed with the fully analytical self-consistent field (SCF) method, the highest resolution integration grids available for density functional theory (DFT), and with the default of level-shifting virtual orbitals disabled. The transferability of the PBF charges from Qsite to Gaussian was tested by comparing the results of vertical excitation calculations in both programs. In one case, vertical excitation energies were calculated with Qsite (B3LYP/LACVP*/OPLS/PFB) and in the other case with ONIOM (B3LYP/LACVP*/Amber/PFB charges). Notwithstanding differences in the level of theory and types of force fields used in the Gaussian and Qsite calculations, excitation energies and oscillator strengths agreed within $\sim 2 \mathrm{~nm}$ and 0.5 units, respectively.

\subsection{Excited States and Electronic Coupling Analysis}

Vertical excitations were computed at the time dependent DFT (TD-DFT) level with the Tamm-Dancoff approximation (TDA). This methodology has been successfully applied previously to predict carotenoid spectra [26]. We were exclusively interested in predicting the transition to the " $1{ }^{1} \mathrm{~B}_{\mathrm{u}}{ }^{+}$" state, which is dominated by single excitation configurations [27]. TD-DFT/TDA is expected to perform well for transitions of this nature, and we consistently reproduced experimental absorption maxima within $0.11 \mathrm{eV}$, which is well within the accepted error for the method [28].

Inter-pigment electronic interactions were investigated through a supramolecular QM/MM or $\mathrm{QM} /$ background electrostatics approach, and the electronic energy transfer methodology implemented in Gaussian 16, which will be referred to hereafter as EET. The EET method $[23,24]$ is a perturbative scheme that calculates the electronic interaction between the transition densities of fragment regions as an approximation to the full QM calculation of the multichromophoric system.

EET was used to compute coupling constants between the Per and Chl $a$ chromophores of PCP at the TDA-B3LYP /6-31g(d) level of theory. The eight Pers and two Chl $a$ molecules in RFPCP were each defined as a fragment for the EET analysis. To perform these calculations in the presence of the PCP complex (protein and lipid molecules) with or without an implicit aqueous solvent, Amber partial atomic charges for the protein and/or PBF charges from the Qsite calculation were added as a background charge distribution. $S_{2}$ excitation wavelengths were obtained from this approach by constructing and diagonalizing a Hamiltonian of the chromophore site energies and coupling 
constants. These wavelengths were directly compared to the wavelengths from the full supramolecular QM calculation that included the Amber and PBF charges as a background electrostatic distribution. Section 3.1 shows the validity of this approach.

In a further calculation that examined the effect of coupling among the Pers on their interaction strength with Chl $a$, the four Pers in each domain of RFPCP were defined as a single fragment which interacted with the $\mathrm{Chl} a$ molecules of the complex. Each Chl $a$ in the two domains was considered a separate fragment.

\subsection{Natural Transition Orbital (NTO) Analysis}

The nature of the computed excitations was inspected using natural transition orbitals $[29,30]$. Predicted absorptions for the pigment assembly were assigned to the chromophore with an NTO hole-particle pair that represented at least $50 \%$ of the character of the excitation. Excitonic states and transition dipole moments for the supramolecular QM/MM calculations will be referred to by these single-chromophore assignments, even though the excitations are delocalized over multiple pigments.

\section{Results}

\subsection{On the Accuracy of Excitonic Coupling Models}

The most accurate description of an electronically interacting assembly of chromophores would, in principle, involve a large-scale QM or QM/MM calculation. While we have carried out such calculations on a single domain of PCP, this approach becomes intractable when considering chromophore interactions between the two protein domains at the TD-DFT level. Here, however, we show that direct calculation of electronic couplings via EET, followed by diagonalization of the exciton Hamiltonian for the four Pers within a single PCP domain gives comparable results to a large-scale calculation in which the QM region contains all four Pers. We find that the excitonic model reproduces well the more accurate supramolecular QM/MM absorption wavelengths with a maximum difference of 2-12 $\mathrm{nm}$ as seen in Table 1. The largest discrepancy occurs under electronic embedding, perhaps reflecting the different polarization responses of four separate versus one large QM region. The overall close agreement of the two approaches attests to the validity of the less computationally demanding fragment-based excitonic model. Thus, we propose that the EET approach can be used to obtain excitation energies for supramolecular assemblies that are too large to be described with a direct QM calculation.

Table 1. Comparison of Per absorption wavelengths simulated with a supramolecular or fragment-based excitonic quantum mechanical (QM) approach in different environments. QM/MM: quantum mechanical/molecular mechanical; EET: electronic energy transfer.

\begin{tabular}{ccccc}
\hline \multirow{2}{*}{ Excitonic State } & \multicolumn{2}{c}{ Vacuum } & \multicolumn{2}{c}{ Solvated PCP Complex } \\
\cline { 2 - 5 } & Supramolecular QM/MM & EET & Supramolecular QM/MM & EET \\
\hline State 1 & 476 & 478 & 502 & 513 \\
State 2 & 469 & 468 & 495 & 501 \\
State 3 & 456 & 458 & 476 & 482 \\
State 4 & 434 & 434 & 447 & 454 \\
\hline
\end{tabular}

All simulated wavelengths have been blue-shifted by $0.11 \mathrm{eV}(\sim 23 \mathrm{~nm})$, which is the discrepancy found for the employed level of theory (Tamm-Dancoff approximation (TDA)-B3LYP/6-31g(d)) relative to experiment for Per in $n$-hexane.

The supramolecular QM/MM calculations also show that the inter-chromophore interactions change the magnitude and direction of the $S_{2}$ transition dipole moments (TDMs; Figure 2), which is reflected in a redistribution of oscillator strength to shorter-wavelength absorption bands as seen in Figure 3. Chromophore coupling also red shifts the lowest-lying Per excitons, thereby helping to close the energetic gap between the Per and Chl $a$ excited states. The EET analysis 
complements these findings by quantifying the pairwise inter-chromophore interactions that are responsible for the wavelength regulation and oscillator strength redistribution predicted in the supramolecular simulation.

Per614
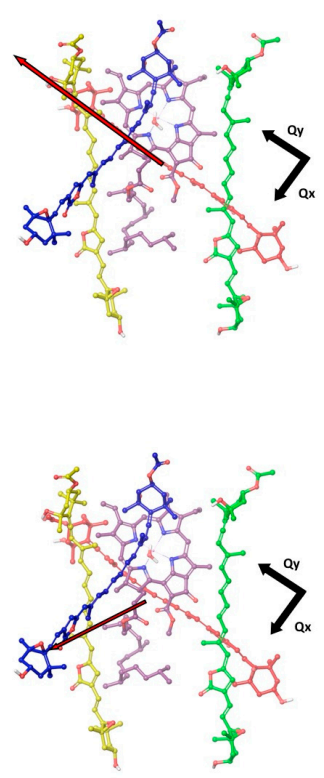

Per611

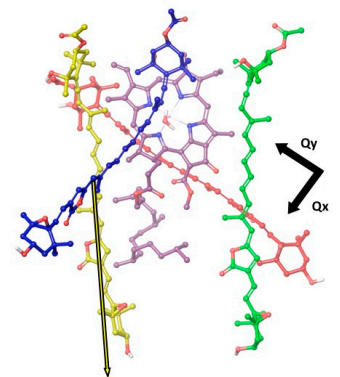

Uncoupled
Per613

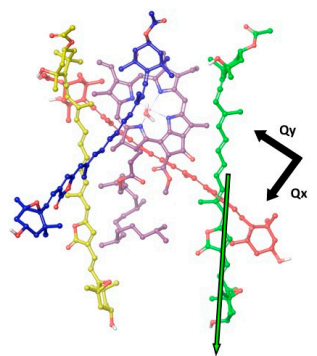

Coupled

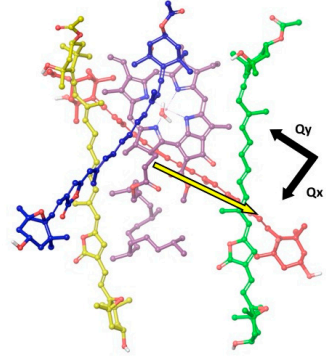

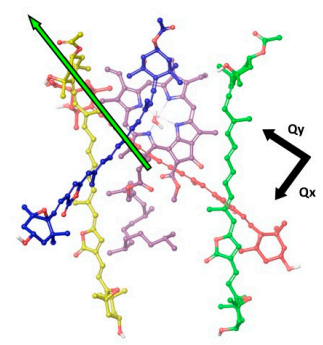

Per612
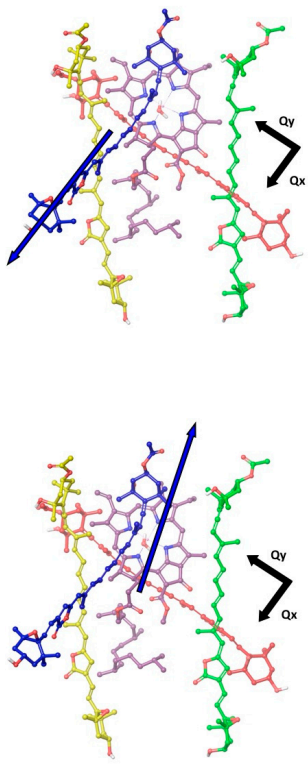

Figure 2. Visualization of the $\mathrm{S}_{0} \rightarrow \mathrm{S}_{2}$ TDMs for (top panel) uncoupled and (bottom panel) coupled Pers in a functional domain of PCP. The coupled states are assigned to the Per that carries at least $50 \%$ of the excitation, and the associated TDM vector is colored to match the given Per. The black vectors point in the directions of the Qy and Qx TDMs of the uncoupled Chl $a$, which are shown in each part of the figure to aid a comparison of the angle between the Per and Chl $a$ TDMs. All TDMs were calculated in the presence of the aqueously solvated PCP complex.

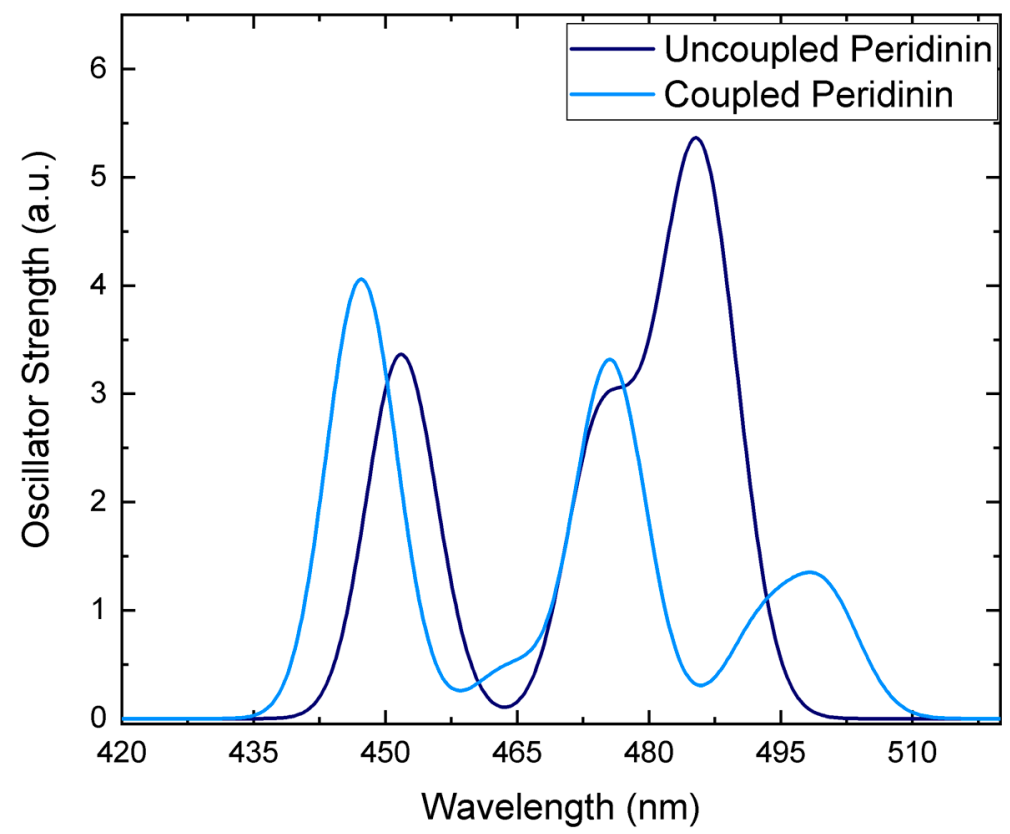

Figure 3. Simulated absorption spectra for the uncoupled (dark blue) and coupled (light blue) four Pers of a cofactor cluster in the solvated PCP complex. Gaussians with a full width at half maximum of $8 \mathrm{~nm}$ were fit to the calculated excitation wavelengths. 
When chromophore electronic coupling is neglected, as shown in the top panel of Figure 2, the TDMs of Per614 and Per612 are nearly aligned to the Chl $a$ Qy and Qx TDMs, respectively. The TDMs of Per611 and Per613 are oriented $37-43^{\circ}$ with respect to the Qy TDM. All the Per TDMs have magnitudes of 17-18 D. When chromophore electronic coupling is introduced among all the Pers in a cofactor cluster, the TDMs for the excitons predominately carried by Per614 and Per612 are $41^{\circ}$ and $28^{\circ}$ respectively from the Qx TDM of Chl $a$. The TDMs for the Per611- and Per613-centered excitations are 20-29 from the Qy TDM of Chl $a$.

The TDM re-orientations induced by chromophore coupling are seemingly consistent with the previous finding that dimeric Per excitons enhance energy transfer to $\mathrm{Chl} a$ relative to monomeric Per excited states [14]. However, the magnitudes of the TDMs are also substantially altered through exciton delocalization. The TDMs for the Per611- and Per614-centered excitations are decreased by 6.9-7.9 D, whereas the TDMs for the Per612- and Per613-centered excitons are increased by 1.7 D. The close proximity of the chromophores further argues against a simple dipole-dipole interaction analysis. Consideration of the interactions between the full transition densities for the separate Pers, or the tetramer of Pers in a cofactor cluster with the central $\mathrm{Chl} a$ gives a different perspective. Individual Pers are found to couple with the Chl Qy state $~ 3-4$ times stronger than the Qx state, whereas the tetramer of electronically interacting Pers couples to both Chl states to comparable extents as seen in Table 2.

Table 2. Comparison of Per-Chl $a$ interaction strengths for uncoupled and coupled Pers in a domain of PCP.

\begin{tabular}{cccc}
\hline $\begin{array}{c}\text { Uncoupled } \\
\text { Chromophores }\end{array}$ & Coupling Constants $\left(\mathbf{c m}^{-\mathbf{1}}\right)$ & Coupled Excitonic State & Coupling Constants $\left(\mathbf{c m}^{-\mathbf{1}}\right)$ \\
\hline Per611-Qy & 298 & Per611 *-Qy & 290 \\
Per612-Qy & 151 & Per612 *-Qy & 48 \\
Per613-Qy & 129 & Per613*-Qy & 121 \\
Per614-Qy & 343 & Per614 *-Qy & 274 \\
Per611-Qx & 67 & Per611*-Qx & 113 \\
Per612-Qx & 27 & Per612 *-Qx & 40 \\
Per613-Qx & 62 & Per613*-Qx & 89 \\
Per614-Qx & 102 & Per614 *-Qx & 113 \\
\hline
\end{tabular}

For the coupled case, an asterisk ${ }^{*}$ ) indicates that the assignment is made to the chromophore with a natural transition orbital (NTO) hole-particle pair that represented at least $50 \%$ of the character of the excitation.

Several interesting conclusions are obtained from the combined analysis of TDM re-orientation and coupling constant changes upon the introduction of Per-Per electronic interactions. (1) There is a general redistribution of coupling strength for all Per-Chl $a$ pairs. Per-Qx interactions gain strength at the expenses of Per-Qy interactions. (2) Upon introducing Per-Per coupling, the TDM of the Per614-based exciton re-aligns roughly in between the polarization axes of Qx and Qy. Considering that Qx state is closest in energy to the longest wavelength absorbing Per614, this alignment and the associated coupling strength redistribution can facility energy transfer from Per614 to Chl $a$. (3) A similar argument can be applied to Per611, which is the second closest Per absorber to the Chl $a$ states. Thus, Per614 and Per611 are likely the sites from which excitation energy most readily flows into the $\mathrm{Chl} a$ states. This conclusion largely confirms the energy transfer model originally proposed by Damjanovic et al. on the basis of Pariser-Parr-Pople calculations [31].

\subsection{Environment Effect on Chromophore Coupling}

We previously found that the electrostatic environment of the aqueously solvated PCP complex significantly contributes to the Per site energy regulation [8]. By varying the energy gaps between individual Pers, and/or screening inter-chromophore interactions, the electrostatic environment could, in principle, also modulate the Per-Per and Per-Chl $a$ interaction strengths [32]. Alternatively, the close packing of PCP cofactors could isolate the chromophores from environmental effects [32]. 
We demonstrated elsewhere that static charge interactions of the Pers with the PCP environment and with one another exert opposing influences on the absorption spectrum [8]. We therefore wondered if or how the environment tunes the inter-chromophore coupling strengths.

Table 3 reports the coupling constants for the cofactor assembly of four Pers (1) isolated in a vacuum, as well as within the (2) vacuum and (3) aqueously solvated PCP complex. Chl $a$ was not included in these calculations, except as part of the electrostatic environment of the PCP complex. Since intra-domain Per-Per electronic interactions have some of the strongest coupling strengths in PCP, as demonstrated below, we anticipate that any solvent-mediated effect should be maximal, and most discernable, for these interactions. The data suggests that the electrostatic PCP environment has a relatively minor (15-42 $\left.\mathrm{cm}^{-1}\right)$ influence on inter-chromophore coupling strengths. For comparison, the environment modulates the Per site energies by up to $700 \mathrm{~cm}^{-1}(17 \mathrm{~nm})$ and increases the overall spectral spread of Per absorptions by a similar amount. This observation implies that the primary or most direct way in which the environment influences inter-chromophore coupling concerns the particular geometric arrangement established for the pigments in the complex. It is an intriguing observation that the four Pers of a PCP functional domain have a common directionality, with the $\gamma$-butenolide end of these chromophores oriented towards the solvent-exposed portal of the complex.

Table 3. Comparison of Per-Per interaction strengths in different environments.

\begin{tabular}{cccc}
\hline \multirow{2}{*}{ Coupled Fragments } & \multicolumn{3}{c}{ Coupling Constants $\left(\mathbf{c m}^{\mathbf{- 1}}\right)$} \\
\cline { 2 - 4 } & Vacuum & Vacuum PCP & Solvated PCP \\
\hline Per611-Per612 & 651 & 609 & 620 \\
Per611-Per613 & 311 & 302 & 278 \\
Per611-Per614 & 379 & 372 & 367 \\
Per612-Per613 & 372 & 365 & 353 \\
Per612-Per614 & 203 & 200 & 189 \\
Per613-Per614 & 473 & 487 & 468 \\
\hline
\end{tabular}

\subsection{Relating Coupling Constants to Experiment}

We present in Table 4 the inter-chromophore coupling constants greater than $100 \mathrm{~cm}^{-1}$. The obtained pairwise inter-pigment coupling strengths are in general agreement with previous reports [13-15,21,26,31]. Per-Per interactions within the same cofactor cluster tend to be the strongest, with coupling constants of 194-619 $\mathrm{cm}^{-1}$. Intra-cluster Per-Chl interactions are competitive with the weakest intra-domain Per-Per interactions $\left(60-343 \mathrm{~cm}^{-1}\right.$ for Pers not coupled with one another). Inter-domain Per-Per, Per-Chl, and Chl-Chl interactions tend to be $<100 \mathrm{~cm}^{-1}$, except for a few noteworthy cases. The four possible pairwise interactions of Per612 and Per613 with Per612' and Per613' (where the primes indicate the $C_{2}$-symmetry related chromophores) have strengths of $255-351 \mathrm{~cm}^{-1}$. Among the weakest chromophore coupling interactions, we find that the coupling constant for the Qy states of the two Chls is $15 \mathrm{~cm}^{-1}$, in excellent agreement with the experimental estimate of $7 \mathrm{~cm}^{-1}$ [33]. This observation indicates that our calculations describe inter-domain interactions remarkably well.

Table 4. Inter-chromophore coupling constants in PCP for interactions larger than $100 \mathrm{~cm}^{-1}$.

\begin{tabular}{cccc}
\hline Chromophore Pair & Coupling Constant & Chromophore Pair & Coupling Constant \\
\hline Per611-Per612 & 619 & Per612-Per613' $^{\prime}$ & 262 \\
Per613-Per614 & 470 & Per613-Per612' $^{\prime}$ & 262 \\
Per612-Per613 & 377 & Per613-Per613' & 255 \\
Per611-Per614 & 365 & Per612-Per614 & 194 \\
Per612-Per612 & 351 & Per612-Qy & 151 \\
Per614-Qy & 343 & Per613-Qx & 129 \\
Per611-Per613 & 310 & Per614-Qx & 102 \\
Per611-Qy & 298 & & \\
\hline
\end{tabular}


The finding that a few inter-domain coupling constants are competitive in strength with intra-domain chromophore interactions raises an interesting question. If chromophore coupling within a cofactor cluster significantly modulates Per site energies in order to extend the spectral range harvested by the complex, as seen in Figure 3, how do inter-domain interactions further refine the absorption spectrum?

It is not computationally tractable to extend the high-level theory region of our multichromophoric QM/MM calculations over both cofactor clusters using TD-DFT. However, we were able to perform calculations with full active space configuration interaction with singles (CIS). Figure 4 compares the simulated spectra for the electronically coupled four Pers in a cofactor cluster of the solvated complex (Per tetramer) at the TD-DFT and CIS levels, and all eight Pers in the functional domains of the RFPCP homodimer (Per octamer) treated with CIS.

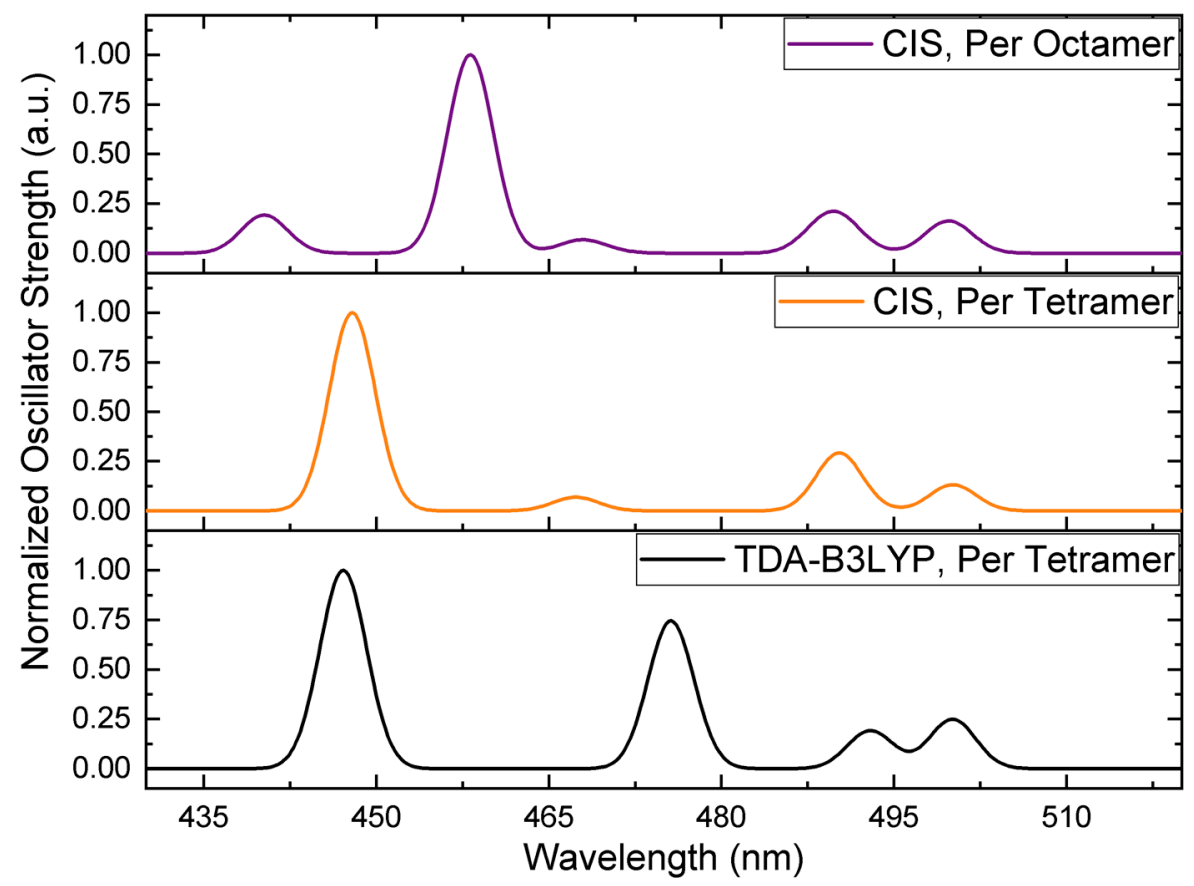

Figure 4. Simulated spectra for Per tetramers at the time dependent density functional theory (TD-DFT) (black) and configuration interaction with singles (CIS) (orange) levels of theory, and an octamer of Pers treated with CIS (purple), within the aqueously solvated PCP complex. All CIS wavelengths were systematically shifted by $0.671 \mathrm{eV}$ to align the longest wavelength absorption with the corresponding TD-DFT band in order to aid a comparison. Gaussians with a full width at half maximum of $4 \mathrm{~nm}$ were fit to the calculated excitation wavelengths.

There are two striking features of Figure 4. First, the shortest and two longest wavelength absorption bands predicted for the Per tetramer at the TD-DFT and CIS levels are essentially identical. The most significant discrepancy in wavelength position $(\sim 10 \mathrm{~nm})$ and relative intensity is found for the feature in the 460-480 $\mathrm{nm}$ region. The level of agreement is nonetheless acceptable.

The second important aspect of Figure 4 is that the spectrum for the Per tetramer and octamer are largely the same. However, the shortest wavelength band is split into features at shorter and longer wavelengths, thereby producing a spectrum with five Per absorptions. Interestingly, a fifth Per absorption was experimentally reported as contributing to the spectrum of the MFPCP complex [18], but the mechanistic origin of this feature, and the responsible chromophore, have yet to be identified. While it may be inferred that the electrostatic inhomogeneity of the $\mathrm{N}$ - and C-terminal domains of MFPCP — the two halves of the protein only share $~ 56 \%$ sequence identity [7]—is responsible for breaking the degeneracy of one of the symmetrically positioned pairs of Pers, our calculations show 
that inter-domain excitonic coupling in the absence of electrostatic differences between protein domains can produce a fifth absorption in the PCP spectrum.

One potential complication with the analysis is that the fifth absorption predicted for RFPCP was not found by spectral reconstruction for this complex [4]. However, spectral reconstruction procedures find the minimum number of absorption bands needed to approximately reproduce a spectrum, and the simulated shortest wavelength absorption for the Per octamer is not particularly intense. It is conceivable that the different electrostatic environment of MFPCP versus RFPCP works to distribute more oscillator strength into this Per $\mathrm{S}_{2}$ exciton. Thus, we hypothesize that the hitherto unexplained fifth short wavelength Per absorption found in the MFPCP spectrum originates from inter-domain excitonic coupling, and may gain oscillator strength under the specific electrostatic influence of the MFPCP complex. This hypothesis is in line with the finding of Carbonera et al. [13] that inter-cluster pigment interactions are necessary to accurately reproduce the circular dichroism spectrum of MFPCP. We are currently investigating our conjecture.

\section{Conclusions}

We have used supramolecular QM/MM calculations to obtain excitation energies and coupling constants with multichromophoric QM regions. Several salient aspects emerge from our calculations. We showed that the EET methodology can be used to obtain quality excitation energies for cases where the inclusion of all PCP cofactors is intractable within QM/MM at a TD-DFT level. Instead, EET can provide coupling constants which are then used to diagonalize the exciton Hamiltonian.

Intra-domain electronic coupling has a substantial effect on the $S_{0} \rightarrow S_{2}$ TDMs of each chromophore unit. Moreover, it appears that coupling aligns the TDMs of particular Per-centered excitons in a manner to favor interaction with both the Qy and Qx states of Chl $a$. We suggest that energy transfer to Chl $a$ is mostly from Per614 and Per611. We also showed that the electrostatic environmental, including an implicit aqueous solvent, has a negligible effect on the strength of chromophore couplings, despite being important in the tuning of site energies. Finally, we put forward a hypothesis to explain the origin of the fifth Per absorption peak in the spectrum of MFPCP. We propose that this peak originates by the inter-domain excitonic coupling between the shortest absorber Per612 and its $\mathrm{C}_{2}$-pseudosymmetric counterpart.

Author Contributions: Conceptualization, M.J.G.-P. and J.A.G.; methodology, M.J.G.-P. and J.A.G.; software, M.J.G.-P.; validation, M.J.G.-P.; formal analysis, M.J.G.-P. and J.A.G.; investigation, M.J.G.-P.; resources, M.J.G.-P.; data curation, M.J.G.-P.; writing—original draft preparation, M.J.G.-P.; writing—review and editing, M.J.G.-P. and J.A.G.; visualization, M.J.G.-P. and J.A.G.; supervision, J.A.G.; project administration, J.A.G.

Funding: This research was funded by the National Science Foundation via a graduate fellowship [DGE-1247393] to M.J.G.-P.

Conflicts of Interest: The authors declare no conflict of interest.

\section{References}

1. Scholes, G.D.; Fleming, G.R.; Olaya-Castro, A.; Van Grondelle, R. Lessons from nature about solar light harvesting. Nat. Chem. 2011, 3, 763-774. [CrossRef] [PubMed]

2. Lewis, N.S.; Nocera, D.G. Powering the planet: Chemical challenges in solar energy utilization. Proc. Natl. Acad. Sci. USA 2006, 103, 15729-15735. [CrossRef] [PubMed]

3. Mirkovic, T.; Ostroumov, E.E.; Anna, J.M.; van Grondelle, R.; Scholes, G.D. Light absorption and energy transfer in the antenna complexes of photosynthetic organisms. Chem. Rev. 2016, 117, 249-293. [CrossRef] [PubMed]

4. Schulte, T.; Niedzwiedzki, D.M.; Birge, R.R.; Hiller, R.G.; Polívka, T.; Hofmann, E.; Frank, H.A. Identification of a single peridinin sensing Chl- $a$ excitation in reconstituted PCP by crystallography and spectroscopy. Proc. Natl. Acad. Sci. USA 2009, 106, 20764-20769. [CrossRef] [PubMed]

5. Croce, R.; Van Amerongen, H. Natural strategies for photosynthetic light harvesting. Nat. Chem. Biol. 2014, 10, 492-501. [CrossRef] [PubMed] 
6. Bautista, J.A.; Hiller, R.G.; Sharples, F.P.; Gosztola, D.; Wasielewski, M.; Frank, H.A. Singlet and triplet energy transfer in the peridinin-chlorophyll $a$-protein from amphidinium carterae. J. Phys. Chem. A 1999, 103, 2267-2273. [CrossRef]

7. Hofmann, E.; Wrench, P.M.; Sharples, F.P.; Hiller, R.G.; Welte, W.; Diederichs, K. Structural basis of light harvesting by carotenoids: Peridinin-chlorophyll-protein from. Science 1996, 272, 1788-1791. [CrossRef] [PubMed]

8. Guberman-Pfeffer, M.J.; Greco, J.A.; Birge, R.R.; Frank, H.A.; Gascón, J.A. Light harvesting by equally contributing mechanisms in a photosynthetic antenna protein. J. Phys. Chem. Lett. 2018, 9, 563-568. [CrossRef] [PubMed]

9. Hudson, B.S.; Kohler, B.E. A low-lying weak transition in the polyene $\alpha$, $\omega$-diphenyloctatetraene. Chem. Phys. Lett. 1972, 14, 299-304. [CrossRef]

10. Fiedor, L.; Fiedor, J.; Pilch, M. Effects of molecular symmetry on the electronic transitions in carotenoids. J. Phys. Chem. Lett. 2016, 7, 1821-1829. [CrossRef] [PubMed]

11. Song, P.-S.; Koka, P.; Prezelin, B.B.; Haxo, F.T. Molecular topology of the photosynthetic light-harvesting pigment complex, peridinin-chlorophyll a-protein, from marine dinoflagellates. Biochemistry 1976, 15, 4422-4427. [CrossRef] [PubMed]

12. Pilch, M.; Pawlikowski, M. Circular dichroism (CD) study of peridinin-chlorophyll a protein (PCP) complexes from marine dinoflagellate algae The tetramer approach. J. Chem. Soc. Faraday Trans. 1998, 94, 227-232. [CrossRef]

13. Carbonera, D.; Giacometti, G.; Segre, U.; Hofmann, E.; Hiller, R.G. Structure-based calculations of the optical spectra of the light-harvesting peridinin-chlorophyll-protein complexes from Amphidinium carterae and Heterocapsa pygmaea. J. Phys. Chem. B 1999, 103, 6349-6356. [CrossRef]

14. Bricker, W.P.; Lo, C.S. Efficient pathways of excitation energy transfer from delocalized $S 2$ excitons in the peridinin-chlorophyll a-protein complex. J. Phys. Chem. B 2015, 119, 5755-5764. [CrossRef] [PubMed]

15. Meneghin, E.; Volpato, A.; Cupellini, L.; Bolzonello, L.; Jurinovich, S.; Mascoli, V.; Carbonera, D.; Mennucci, B.; Collini, E. Coherence in carotenoid-to-chlorophyll energy transfer. Nat. Commun. 2018, 9, 3160. [CrossRef] [PubMed]

16. Roscioli, J.D.; Ghosh, S.; LaFountain, A.M.; Frank, H.A.; Beck, W.F. Quantum coherent excitation energy transfer by carotenoids in photosynthetic light harvesting. J. Phys. Chem. Lett. 2017, 8, 5141-5147. [CrossRef] [PubMed]

17. Ghosh, S.; Bishop, M.M.; Roscioli, J.D.; LaFountain, A.M.; Frank, H.A.; Beck, W.F. Excitation energy transfer by coherent and incoherent mechanisms in the peridinin-chlorophyll a protein. J. Phys. Chem. Lett. 2017, 8, 463-469. [CrossRef] [PubMed]

18. Ilagan, R.P.; Shima, S.; Melkozernov, A.; Lin, S.; Blankenship, R.E.; Sharples, F.P.; Hiller, R.G.; Birge, R.R.; Frank, H.A. Spectroscopic properties of the main-form and high-salt peridinin- chlorophyll a proteins from Amphidinium carterae. Biochemistry 2004, 43, 1478-1487. [CrossRef] [PubMed]

19. Sastry, G.M.; Adzhigirey, M.; Day, T.; Annabhimoju, R.; Sherman, W. Protein and ligand preparation: Parameters, protocols, and influence on virtual screening enrichments. J. Comput. Aided Mol. Des. 2013, 27, 221-234. [CrossRef] [PubMed]

20. Tannor, D.J.; Marten, B.; Murphy, R.; Friesner, R.A.; Sitkoff, D.; Nicholls, A.; Honig, B.; Ringnalda, M.; Goddard, W.A., III. Accurate first principles calculation of molecular charge distributions and solvation energies from ab initio quantum mechanics and continuum dielectric theory. J. Am. Chem. Soc. 1994, 116, 11875-11882. [CrossRef]

21. Bricker, W.P.; Lo, C.S. Excitation energy transfer in the peridinin-chlorophyll a-protein complex modeled using configuration interaction. J. Phys. Chem. B 2014, 118, 9141-9154. [CrossRef] [PubMed]

22. Frisch, M.J.; Trucks, G.W.; Schlegel, H.B.; Scuseria, G.E.; Robb, M.A.; Cheeseman, J.R.; Scalmani, G.; Barone, V.; Petersson, G.A.; Nakatsuji, H.; et al. Gaussian 16, Revision B.01; Gaussian Inc.: Wallingford, CT, USA, 2016.

23. Curutchet, C.; Mennucci, B. Toward a molecular scale interpretation of excitation energy transfer in solvated bichromophoric systems. J. Am. Chem. Soc. 2005, 127, 16733-16744. [CrossRef] [PubMed]

24. Russo, V.; Curutchet, C.; Mennucci, B. Towards a molecular scale interpretation of excitation energy transfer in solvated bichromophoric systems. II. the through-bond contribution. J. Phys. Chem. B 2007, 111, 853-863. [PubMed] 
25. Murphy, R.B.; Philipp, D.M.; Friesner, R.A. A mixed quantum mechanics/molecular mechanics (QM/MM) method for large-scale modeling of chemistry in protein environments. J. Comp. Chem. 2000, 21, 1442-1457. [CrossRef]

26. Andreussi, O.; Knecht, S.; Marian, C.M.; Kongsted, J.; Mennucci, B. Carotenoids and light-harvesting: From DFT/MRCI to the tamm-dancoff approximation. J. Chem. Theory Comput. 2015, 11, 655-666. [CrossRef] [PubMed]

27. Knecht, S.; Marian, C.M.; Kongsted, J.; Mennucci, B. On the photophysics of carotenoids: A multireference DFT study of peridinin. J. Phys. Chem. B 2013, 117, 13808-13815. [CrossRef] [PubMed]

28. Adamo, C.; Jacquemin, D. The calculations of excited-state properties with Time-Dependent Density Functional Theory. Chem. Soc. Rev. 2013, 42, 845-856. [CrossRef] [PubMed]

29. Martin, R.L. Natural transition orbitals. J. Chem. Phys. 2003, 118, 4775-4777. [CrossRef]

30. Dreuw, A.; Head-Gordon, M. Single-reference ab initio methods for the calculation of excited states of large molecules. Chem. Rev. 2005, 105, 4009-4037. [CrossRef] [PubMed]

31. Damjanović, A.; Ritz, T.; Schulten, K. Excitation transfer in the peridinin-chlorophyll-protein of Amphidinium carterae. Biophys. J. 2000, 79, 1695-1705. [CrossRef]

32. Mennucci, B.; Curutchet, C.; Cammi, R.; Scholes, G.D. How the molecular environment controls excitation energy transfer and light harvesting: A quantum mechanical model. AIP Conf. Proc. 2007, 963, 346. [CrossRef]

33. Kleima, F.J.; Wendling, M.; Hofmann, E.; Peterman, E.J.; van Grondelle, R.; van Amerongen, H. Peridinin chlorophyll a protein: Relating structure and steady-state spectroscopy. Biochemistry 2000, 39, 5184-5195. [CrossRef] [PubMed]

Sample Availability: Coordinates of all the calculated structures are available from the authors.

(C) 2018 by the authors. Licensee MDPI, Basel, Switzerland. This article is an open access article distributed under the terms and conditions of the Creative Commons Attribution (CC BY) license (http://creativecommons.org/licenses/by/4.0/). 